\title{
Skin inflammation activates intestinal stromal fibroblasts and promotes colitis
}

\author{
Tatsuya Dokoshi, Jason S. Seidman, ${ }^{2}$ Kellen J. Cavagnero, ${ }^{1}$ Fengwu Li, ${ }^{1}$ Marc C. Liggins, ${ }^{1}$ Bryn C. Taylor, ${ }^{3}$ Jocelyn Olvera, ${ }^{4}$ \\ Rob Knight, ${ }^{5}$ John T. Chang, ${ }^{4}$ Nita H. Salzman, ${ }^{6}$ and Richard L. Gallo ${ }^{1}$
}

'Department of Dermatology, ${ }^{2}$ Department of Cellular and Molecular Medicine, ${ }^{3}$ Biomedical Sciences Graduate Program, ${ }^{4}$ Department of Medicine, and ${ }^{5}$ Department of Pediatrics, UCSD, La Jolla, California, USA. ${ }^{6}$ Departments of Pediatrics, Microbiology, and Immunology, Medical College of Wisconsin, Milwaukee, Wisconsin, USA.

\begin{abstract}
Inflammatory disorders of the skin are frequently associated with inflammatory bowel diseases (IBDs). To explore mechanisms by which these organs communicate, we performed single-cell RNA-Seq analysis on fibroblasts from humans and mice with IBD. This analysis revealed that intestinal inflammation promoted differentiation of a subset of intestinal stromal fibroblasts into preadipocytes with innate antimicrobial host defense activity. Furthermore, this process of reactive adipogenesis was exacerbated if mouse skin was inflamed as a result of skin wounding or infection. Since hyaluronan (HA) catabolism is activated during skin injury and fibroblast-to-adipocyte differentiation is dependent on $\mathrm{HA}$, we tested the hypothesis that HA fragments could alter colon fibroblast function by targeted expression of human hyaluronidase- 1 in basal keratinocytes from mouse skin. Hyaluronidase expression in the skin activated intestinal stromal fibroblasts, altered the fecal microbiome, and promoted excessive reactive adipogenesis and increased inflammation in the colon after challenge with dextran sodium sulfate. The response to digested HA was dependent on expression of TLR4 by preadipocytes. Collectively, these results suggest that the association between skin inflammation and IBD may be due to recognition by mesenchymal fibroblasts in the colon of HA released during inflammation of the skin.
\end{abstract}

\section{Introduction}

All human epithelial surfaces use innate host defense systems to detect and appropriately respond to environmental challenges such as physical injury and bacterial invasion. Skin and the intestine cope with their very different environments with distinct innate immune strategies to defend against unique pathogens. However, despite their differences, inflammatory disorders of these critical barrier organs are frequently observed together (14). For example, patients with inflammatory bowel disease (IBD) have a higher risk of inflammatory skin diseases (5), and patients with primary skin diseases also have a higher risk of IBD (6). These observations have led to speculation that communication occurs between these epithelial surfaces, a phenomenon that has also been observed with asthma, food allergy, and atopic dermatitis (7). Although a mechanism responsible for such immune crosstalk remains largely unknown, it has been hypothesized that a signal exists between epithelial organs that results in the disruption of immune homeostasis at distant epithelial surfaces.

Host defense of both the skin and gut involves multiple systems that include the action of classical immunocytes as well as innate antimicrobial effector molecules (8). Recent studies

Conflict of interest: RLG is a cofounder of, scientific advisor and consultant for, and has equity in MatriSys Biosciences; and is a consultant for, receives income from, and has equity in Sente.

Copyright: (5) 2021, American Society for Clinical Investigation.

Submitted: January 12, 2021; Accepted: September 16, 2021; Published: November 1, 2021.

Reference information: J Clin Invest. 2021;131(21):e147614.

https://doi.org/10.1172/JCl147614. have revealed that the heterogeneous population of fibroblasts in both the skin dermis and in the submucosal layer of the intestine contributes to immune defense (9-12). These molecules support the development of immunocytes and provide innate immunoprotective functions when they are triggered to undergo adipogenic differentiation by local injury or infection. These latter defensive functions of preadipocyte fibroblasts (pADs) are distinct from the roles of mature adipocytes (ADs), which have independent inflammation-promoting functions $(13,14)$. The process of preadipocyte maturation has long been observed in IBD as the phenomenon of "creeping fat," but the role of the preadipocyte as an immune fibroblastic cell (IFC) in host defense of the colon is not understood.

The differentiation of pADs to ADs is dependent on the presence of hyaluronan (HA) in the extracellular matrix $(15,16)$. HA has several immunoregulatory functions (17-19). In response to inflammation, HA is digested into low-molecular-weight fragments, with unique immunologic activity as a damage-associated molecular pattern (DAMP; refs. 20-22). In this study we sought to understand whether fibroblasts participate in the pathogenesis of IBD and if HA could act as a signal from the skin to alter intestinal immune function through effects on fat cell differentiation. Our observations provide evidence that advances understanding of the pathophysiology behind the association of skin and intestinal disease.

\section{Results}

To begin to evaluate the potential role of fibroblasts in inflammatory bowel disease (IBD), we reanalyzed currently available single-cell RNA-Seq (scRNA-Seq) data from colons of healthy 
A Human colon fibroblast cluster

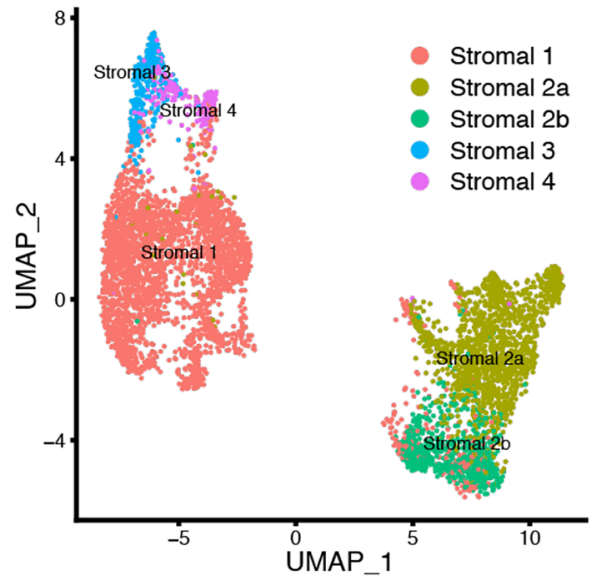

D

GO terms: sample markers

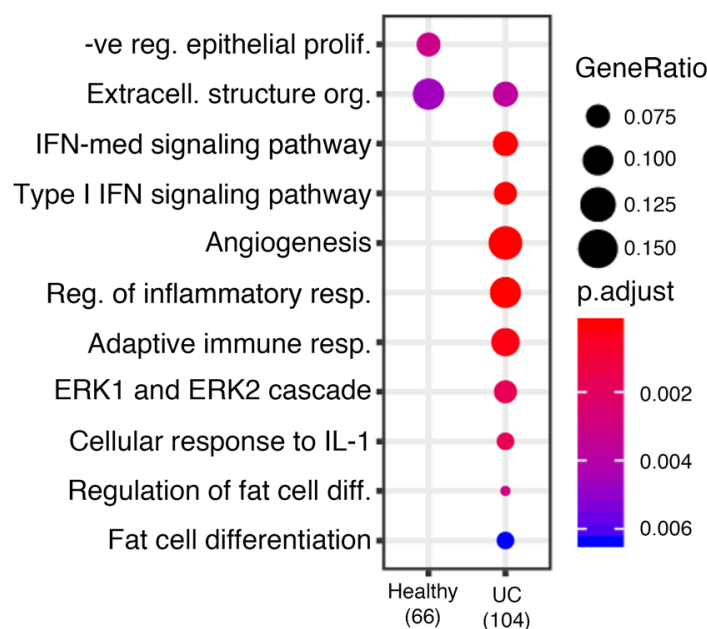

B

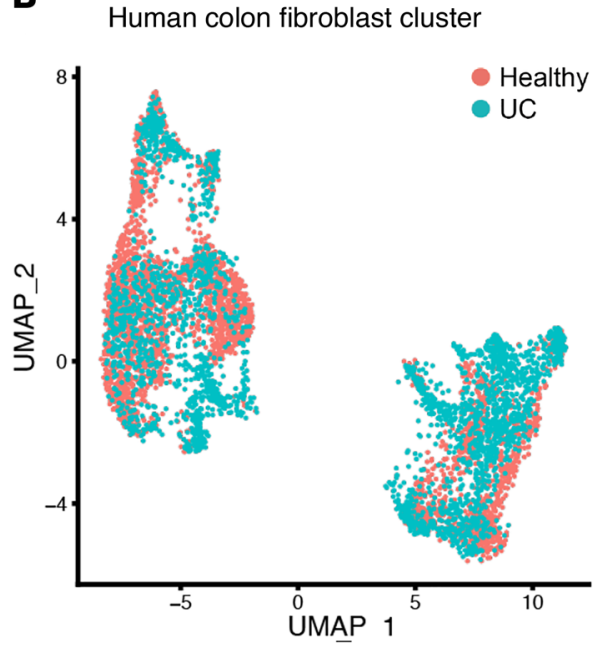

C

Human colon fibroblast

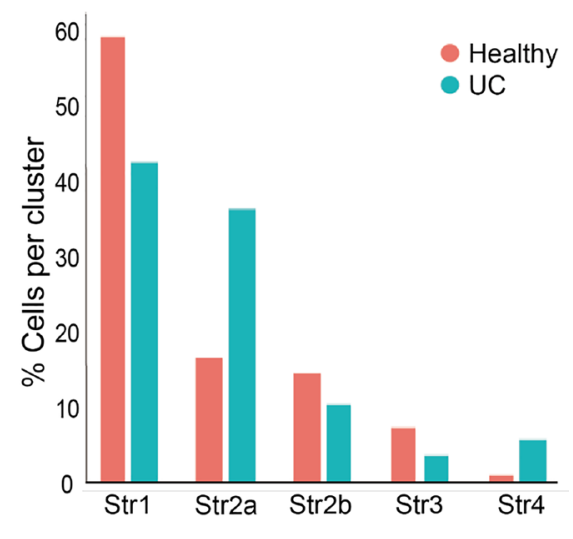

E

GO terms: cluster markers

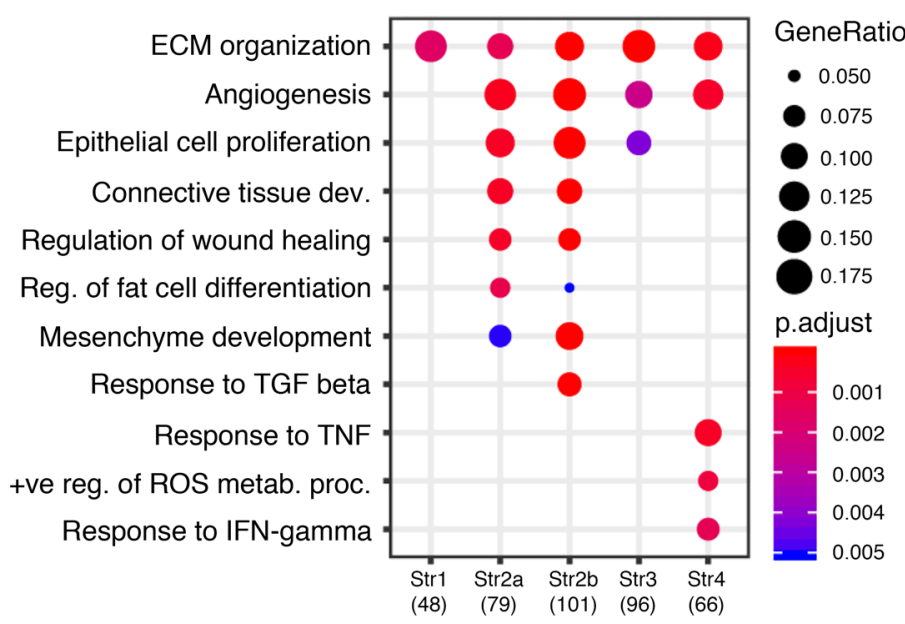

Figure 1. Transcriptional evidence of adipogenesis in human IBD. UMAP plots of PDCFRA-positive cells in patients with UC and healthy individuals, colored by cluster (A) and by the presence of disease (B). (C) Relative abundance of each cluster. (D and $\mathbf{E})$ Selected GO terms showing enrichment among top marker genes and their clusters. -ve, negative; reg., regulation; extracell., extracellular; prolif., proliferation; org., organization; med, mediated; resp., response; diff., differentiation; dev., development; +ve, positive; metab., metabolic; proc., processes; Str, stromal.

individuals and patients with newly diagnosed ulcerative colitis (UC) (Gene Expression Omnibus [GEO] database GSE95459 and GSE114374). Interrogation of the transcriptional response of stromal fibroblasts was performed based on the expression of plateletderived growth factor receptor $\alpha$ (PDGFRA). PDGFRA-positive stromal fibroblasts from these donors subclustered into 5 distinct populations (Figure 1A). Patients with UC had an increase in clusters 2a and 4 and a decrease in clusters 1 and 3 (Figure 1, B and C). Gene ontogeny (GO) analysis revealed that colon fibroblasts in patients with UC had increased expression of genes involved in fat cell differentiation and inflammation and decreased expression of genes involved in extracellular matrix formation (Figure 1, D and E). These results suggested that the expansion of preadipocyte subpopulations of colon fibroblasts was associated with UC, a finding consistent with the mesenteric adipose tissue expansion surrounding inflamed intestine clinically observed on gross examination in Crohn's disease (CD) and known as creeping fat
$(4,10)$. Furthermore, this process of fibroblast differentiation into adipose (reactive adipogenesis) has also been shown to occur in mice in response to intestinal injury by dextran sodium sulfate (DSS) or in the skin after infection by Staphylococcus aureus (23, 24). This response is necessary for immune defense, as inhibition of fibroblast differentiation into adipocytes decreases antimicrobial immune defense and increases bacterial penetration. Therefore, the process of reactive adipogenesis as observed in these individuals with IBD was likely the result of the differentiation into adipocytes of a subset of IFCs that contributes to the defense milieu of the colon.

We next studied whether inflammation localized to the skin could affect the colon of mice and thus potentially model the human clinical association of skin and intestinal disease. To test this, were evaluated 2 different forms of skin injury in mice: full-thickness aseptic incisional wounding (25) and infection by intradermal injection of $S$. aureus (12). Forty-eight hours after 
injury to the skin, the intestine was damaged by addition of DSS to drinking water (26). Compared with control mice, mice with skin wounds showed an increase in intestinal disease severity in response to DSS, with a greater decrease in body weight and a delayed recovery after DSS was withdrawn (Figure 2A). Consistent with this, mice with skin wounds also had greater expression of Il6 in colon tissue (Figure 2B). Adiponectin (Adipoq) mRNA was also increased in the colon of mice with relative to mice without injured skin (Figure 2C), a finding consistent with an increase in adipogenesis in the colon after wounding of the skin. A similar response in the colon was observed in mice with the alternative form of skin inflammation. Mice with $S$. aureus skin infections exhibited a greater loss in body weight and higher levels of Il6 and Adipoq mRNA in the colon after DSS treatment (Figure 2, D-F).

Histological examination of the colon on day 14 after administration of DSS in the presence or absence of skin inflammation showed direct evidence of increased reactive adipogenesis in the colon. Mice with skin inflammation and DSS challenge had greater expansion of the submucosal fat layer in the colon and increased inflammatory activity at the luminal epithelium (Figure $2, G^{-} J$ ). Neither skin wounding nor $S$. aureus infection resulted in weight loss or histologic changes in the colon without DSS administration. These results demonstrated that inflammatory skin injury by wounding or infection can prime the colon to an increased inflammatory response to DSS. In each of these models, intestinal submucosal stromal cells had an increased reactive adipogenic response after skin injury.

To further define how skin injury may influence the function of intestinal submucosal cells, we next performed scRNA-Seq on fibroblasts isolated from mouse colon after skin wounding. To focus on the effects of skin inflammation, we performed this analysis in the absence of colitis. PDGFRA ${ }^{+}$stromal fibroblasts from the colon were distinguishable in multiple cell clusters in all mice (Figure 3A). Comparison of these cell clusters when isolated from mice with and without skin wounds showed that the distribution of cells within these clusters was different after skin injury (Figure $3 \mathrm{~B}$ ). In particular, mice with skin wounds showed an increase in intestinal fibroblasts identified as cluster 5 , and a decrease in cells identified as belonging to clusters 0,2 , and 7 (Figure 3C). GO analysis of all fibroblasts in samples from mice with skin wounds showed that the wounding resulted in increased expression of transcripts associated with a proinflammatory response and fat cell differentiation, as well as loss of transcripts associated with maintenance of the extracellular matrix (Figure 3D). Focused GO analysis of genes in cluster 5 showed that this subset included genes associated with fat cell differentiation (Figure 3E). Pseudotime analysis (27) distinguished 3 lineages within the fibroblast populations and the expression of several genes characteristic of each lineage (Figure 3, F and G). Most notably, after skin wounding we observed that the fibroblasts with the greatest representation of genes associated with fat cell differentiation shifted from cluster 2 toward cluster 5 in lineage 3 (Figure 3H). In addition to the 5 most highly abundant genes found in cluster 5 , CCAAT enhancer binding protein beta $(C e b p b)$ was detected in this subset (Figure 3I). This transcription factor is critical for expression of the cathelicidin antimicrobial peptide (Camp) and is a characteristic gene expressed during reactive adipogenesis $(12,28)$. Overall, the analysis of the transcriptional response of colon submucosal fibroblasts suggested that injury to the skin triggered cells in cluster 5 of the submucosal fibroblast cell community toward a more proinflammatory and adipogenic state.

Zfp423 is expressed in fibroblasts upon initiation of adipogenesis (29). To independently confirm that skin injury can promote reactive adipogenesis in the colon, we subjected $\mathrm{Zfp} 423^{\text {lac } /+}$ reporter mice (30) to skin wounding and DSS challenge. Visualization of lacZ expression with $\beta$-gal demonstrated that skin wounding resulted in increased expression of Zfp423 in the distal colon and mesenchymal fat (Figure 3, J and $\mathrm{K}$ ). These data were consistent with the increased expression of Adipoq mRNA and histological evidence of increased expansion of the adipose layer in the colon after skin wounding and DSS challenge as shown in Figure 2. These results confirmed that skin injury increased the capacity of DSS to promote adipogenesis in the colon.

Next, we examined the effect of skin injury in an alternative model of murine colitis driven by the spontaneous autoimmune inflammation that is observed in $\mathrm{Il1O}$-deficient (IL10 ${ }^{-/}$) mice. Forty-eight hours after skin wounding, IL10 ${ }^{-/}$mice with skin wounds had greater disease severity in the colon compared with IL10 ${ }^{-/-}$mice without skin injury (Figure $4, \mathrm{~A}$ and B). An increase in the expression of Cebpb (Figure $4 \mathrm{C}$ ) and an increase in the number of preadipocyte secreted factor 1-positive (Pref1-positive) cells as an indicator of early adipogenesis (ref. 31 and Figure 4D) was also observed. These results demonstrate that reactive adipogenesis also can occur in the colon of IL10 ${ }^{-/-}$mice and that skin wounding exacerbates colitis in this model.

To determine why injury to the skin can be detected by the submucosal fibroblasts in the colon, we evaluated the trafficking of activated immunocytes from the skin to the colon. To assess this, we used photoconvertible (KikGR) mice, in which all cells can be induced to change from green to red fluorescence with illumination by high-intensity violet light at $435 \mathrm{~nm}(32,33)$. Mice were topically irradiated to label all cells red in the skin and then subjected to skin wounding as performed in the prior experiments. Forty-eight hours after skin injury, the colon was evaluated by FACS analysis for the presence of KikRed cells that would have come from the skin (Supplemental Figure 1, A and B; supplemental material available online with this article; https://doi.org/10.1172/ JCI147614DS1). We detected abundant KikRed cells in the skin after photoconversion, but no evidence of migration of KikRed cells from the skin to the colon was seen 2 days following skin injury or in injured mice with colitis (Supplemental Figure 1C).

HA is necessary for development of preadipocytes $(9,34$, 35). HA is a major component of the skin extracellular matrix $(36,37)$ and following injury or infection is degraded in the skin, where it is converted from its endogenous high-molecular-weight form (HMW-HA) to soluble low-molecular-weight fragments (LMW-HA; ref. 18, 35). We hypothesized that the release of LMW-HA fragments from the skin could enhance the adipogenic response of stromal fibroblasts in the colon. To first test whether this phenomenon can occur in vitro, we isolated LMW-HA from cultured primary mouse fibroblasts ( $\mathrm{mFBs}$ ) overexpressing human hyaluronidase-1 (hHYAL1) and induced to increase HA synthesis by differentiation into adipocytes (35). HMW-HA (500-1000 $\mathrm{kDa}$ ) synthesized during differentiation was digested by hHYAL1 
A

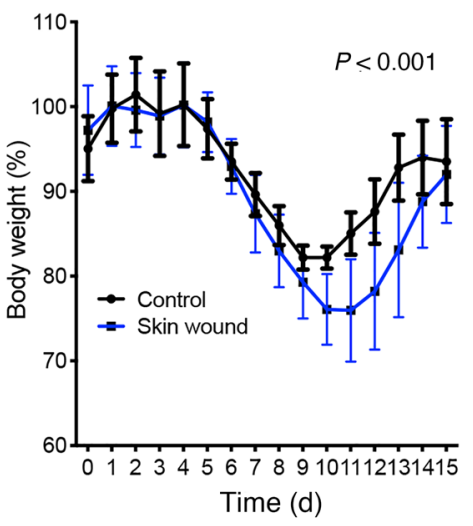

D

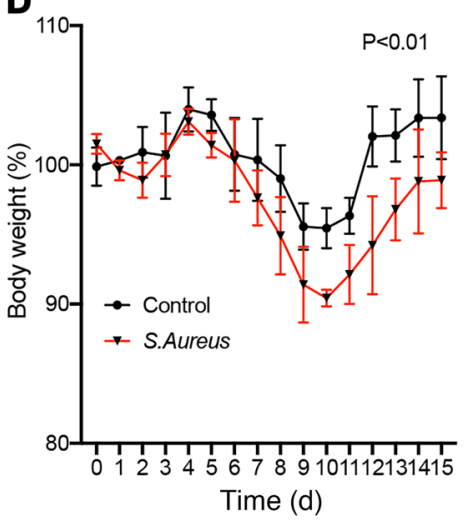

B

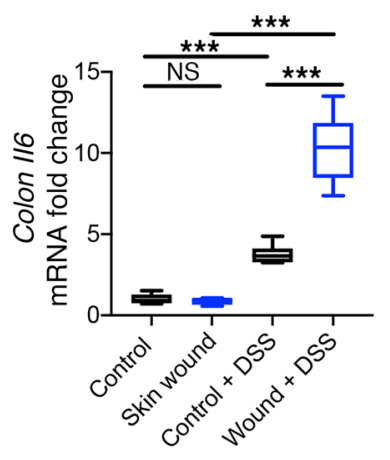

$\mathbf{E}$

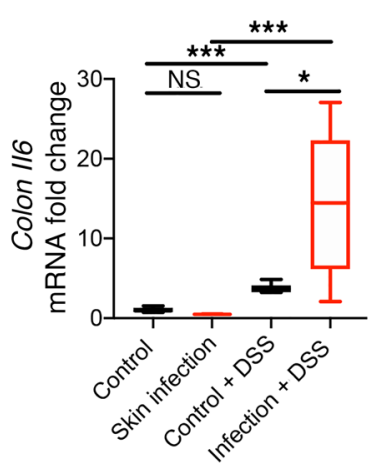

C

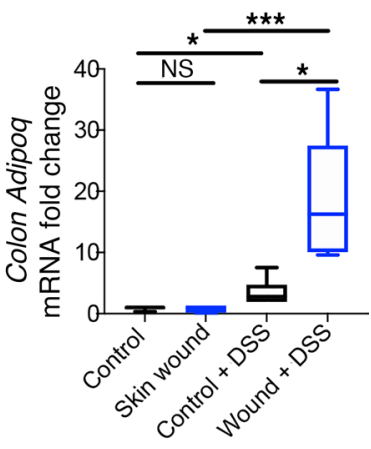

$\mathbf{F}$

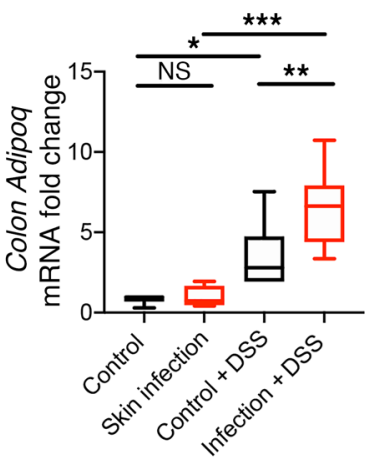

G

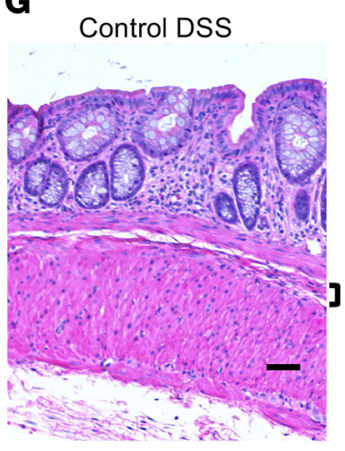

I

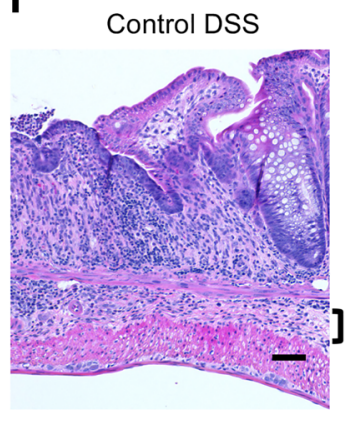

Wound DSS

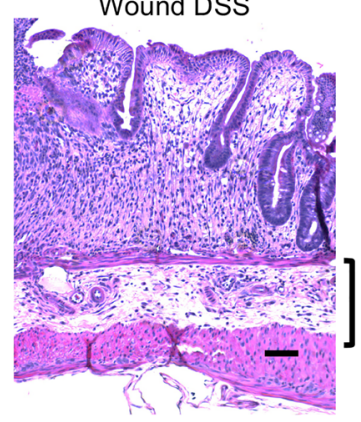

Skin infection DSS

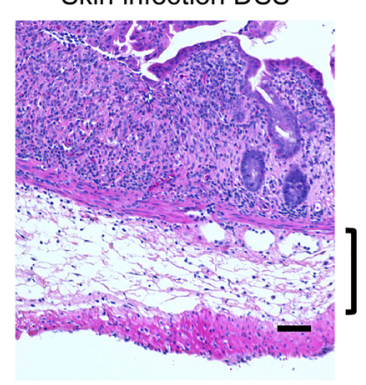

H

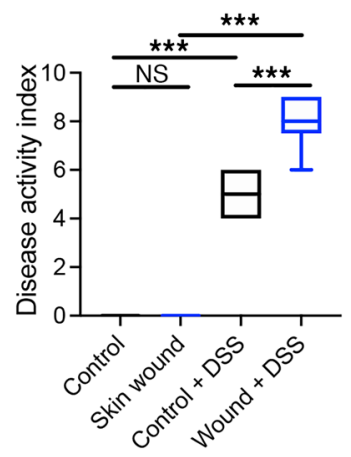

J

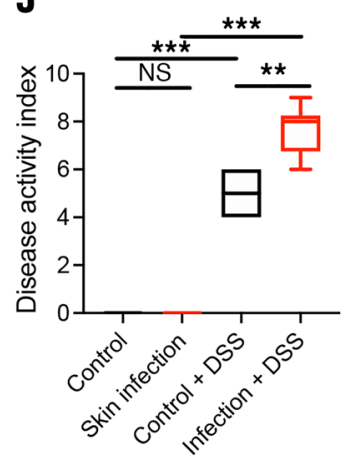

Figure 2. Skin inflammation exacerbates DSS colitis in mice. (A-C, G, and $\mathbf{H})$ Mice received incisional wounds of the skin 48 hours prior to DSS administration. (D-F, I, and J) S. aureus $\left(1 \times 10^{6} \mathrm{CFU}\right)$ was injected intradermally in skin 48 hours prior to DSS. (A and $\left.\mathbf{D}\right)$ Percent change in body weight normalized to weight on day 0. (B, C, E, and F) mRNA expression of $1 / 6$ and Adipoq in the colon. (G and I) Histological images of the colon on day 14 . Tissue was stained with $\mathrm{H \& E}$. Scale bars: $50 \mu \mathrm{m}$. Brackets delineate the submucosal adipose layer. (H and I) Disease activity index based on histological images of colon from mice on day 14. Error bars indicate mean $\pm \mathrm{SEM}$; $P$ values based on 2-way ANOVA with correction for multiple comparison. ${ }^{*} P<0.05$, ${ }^{* *} P<0.01,{ }^{* * *} P<$ 0.001. $n=6$ in all experiments; each experiment was repeated 3 times. 
to LMW-HA (6.8-250 kDa; Supplemental Figure 2). HA fragments generated from these cells were then transferred to naive WT mFBs (Figure 5A). The transfer of LMW-HA to mFBs in the presence of fat cell differentiation medium increased the expression of Pref1 and increased morphologic evidence of adipogenesis as measured by increased lipid staining with Oil Red O (Figure 5, B-D). Furthermore, since CD44, TLR2, and TLR4 have each been reported to be receptors for HA (38-40), we isolated mFBs from mice lacking CD44, TLR2, or TLR4 and tested their responsiveness to HA fragments. TLR4 ${ }^{-/-}$cells did not respond with an increase in Pref1 expression after exposure to LMW-HA (Figure 5B). Lipid accumulation as detected by Oil Red $\mathrm{O}$ was inhibited in both $\mathrm{CD} 44^{-/-}$and TLR $4^{-/-}$cells under differentiation conditions and upon addition of LMW-HA (Figure 5C). These observations supported the hypothesis that binding to HA is important for normal adipogenesis and that the addition of HA fragments can increase this response.

To directly test whether HA digestion in the skin could influence the colon, we next used transgenic mice expressing a tissuespecific inducible expression construct of hHYAL1 (K14/HYAL1). These mice effectively degrade HA in the skin $(34,35)$. K14/HYAL1 mice showed expression of K14 mRNA only in the skin and no evidence of spontaneous inflammation in the colon (Supplemental Figure 3, A-C). Analysis of HA in the colon by detection with hyaluronan binding protein (HABP) showed an increase in HA in the colon of K14-HYAL1 mice (Supplemental Figure 3D). In contrast to that in the colon, HA staining by HABP was decreased in the dermis of K14/HYAL1 mice and in the skin of WT mice with skin wounds (Supplemental Figure 4). This loss of HA staining could reflect the absence of HA or the digestion to LMW-HA, which is not as easily detected by HABP (Supplemental Figure 5).

Despite the lower sensitivity of HABP for LMW-HA, an increase in the relative level of HA was observed in serum in mice with wounding or $S$. aureus infection of the skin and in K14/ HYAL1 mice (Figure 6A). Similar to mice with skin wounds, K14/ HYAL1 mice also had higher expression of Cebpb in the colon (Figure 6B). No change in neutrophils, macrophages, dendritic cells, CD4-positive cells, or CD8-positive cells was detected in the colon of K14/HYAL1 mice (Supplemental Figure 6, A-E). Epithelial integrity as assessed by absorption did not change in intestinal colon of K14/HYAL1 mice or mice with skin wounds that had been orally administered FITC-dextran; however, following challenge with DSS, a significant increase in intestinal permeability was observed in mice with skin wounds and in K14/ HYAL1 mice compared with control mice (Figure 6C). Remarkably, K14/HYAL1 mice showed a great increase in disease severity after challenge by DSS, as demonstrated by increased weight loss and an increase in mortality (Figure 6, D and E). Surviving K14/HYAL1 mice showed expansion of peri-intestinal fat and increased epithelial damage compared with controls (Figure 6, F-H). K14/HYAL1 mice also showed higher expression of Il6 and Adipoq in the colon (Figure 6, I and J).

Additional evidence of enhanced reactive adipogenesis in K14/HYAL1 mice was the large increase in Camp mRNA and Pref1 protein expression after exposure to DSS (Supplemental Figure 7). These findings were similar to that observed in the colon after skin injury. Furthermore, whole-tissue RNA-Seq of the colon submuco- sa in K14/HYAL1 mice identified a broad transcriptional response that included an increase in Pdgrfa mRNA even in the absence of DSS challenge (Supplemental Figure 8).

Finally, given the constitutive changes in the colon of mice with altered skin HA catabolism, we asked whether the skin could influence the fecal microbiome. 16S rRNA amplicon sequencing of feces revealed that the microbiomes of cohoused control and K14/ HYAL1 mice were significantly different (Supplemental Figure 9). As expected, DSS challenge also resulted in a major shift in the fecal microbial community. In the presence of DSS, a difference in microbial composition was not detectable between control and K14/HYAL1 mice. These results suggest that digestion of HA in the skin not only alters the host immune response to DSS in the colon, but also changes the fecal microbial community. This change in the intestinal microbiome could serve as an additional signal to trigger adipogenesis following the loss of epithelial integrity (10).

\section{Discussion}

The results of this study show how damage to the skin influences the function of the colon. Specifically, skin inflammation was shown to stimulate IFC subsets of fibroblasts in the colon submucosa and promote increased adipocyte differentiation during colitis. While multiple mechanisms may contribute to this communication system between epithelial organs, our observations suggest that the digestion of HA that occurs during skin inflammation plays an important role in transmitting a signal of injury from the skin to the colon. Targeted expression of hyaluronidase in mouse skin focused on this unique immune signaling system, since HYAL1 expression did not induce inflammation in the skin but recapitulated the large effect of a skin-specific intervention on the inflammatory phenotype of the colon. As the action of HA included amplification of reactive adipogenesis that was also seen in human subjects with IBD, the process of dermal HA catabolism may provide an explanation for why inflammatory skin disorders are frequently associated with IBD.

No change in the abundance of immunocytes in the colon was detected after skin injury or HA digestion in the skin. This does not eliminate the possibility that skin hyaluronidase expression could result in undetected changes in lymphoid subpopulations, circulating cytokines, or hormonal signals that could influence the colon. Indeed, an overall change in the host defense status of the colon appears probable given the change seen in the fecal microbiome upon expression of hyaluronidase in skin. This constitutive change may then contribute to the enhanced susceptibility to colitis following DSS challenge. The observed contribution of the local tissue microbial communities, and other immune signals, is a subject of ongoing investigations but does not diminish the evidence that HA fragments from the skin are an important element that influences the phenotype observed in the colon.

HA was previously known to be digested following local injury or inflammation and to act as a DAMP (40), but the consequences of that response were not well understood. In models of both lung and skin injury, TLR4 has been shown to recognize fragments of HA as an inflammatory mediator (41-43). Conversely, intact HMW-HA is also important for cell migration and development, including during fat cell development $(9,35$, $44,45)$. Thus, previous investigations into HA catabolism have focused on local cell events rather than a distal response. Our 
A Mouse colon fibroblast cluster

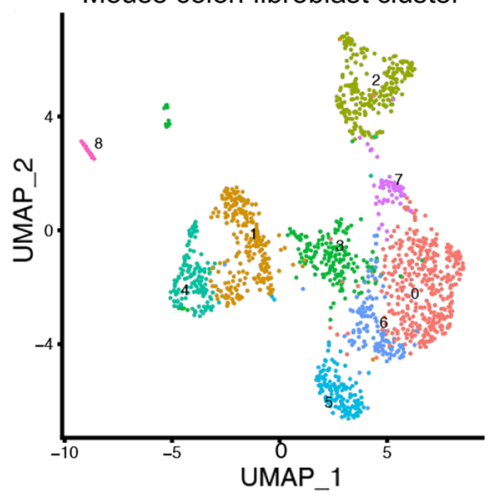

D

GO terms: sample markers

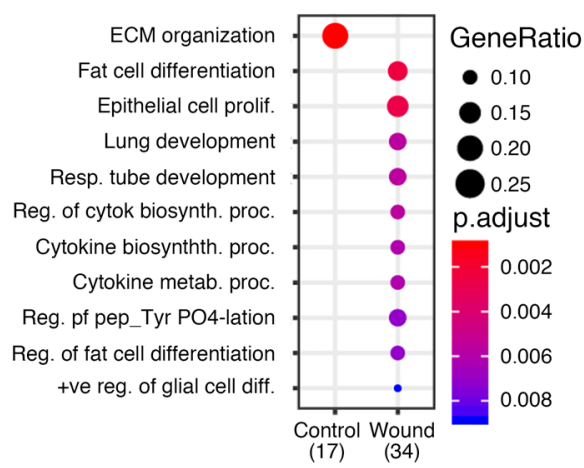

G

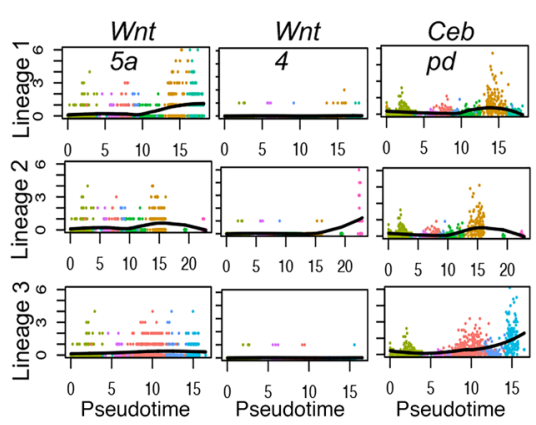

I

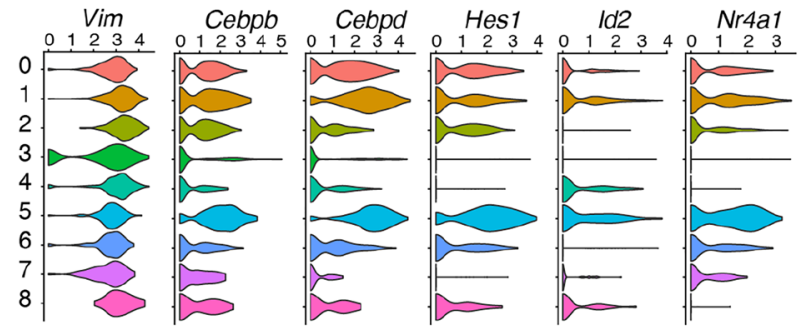

H
B

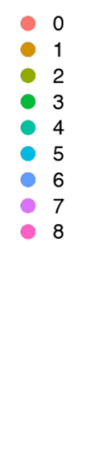

E

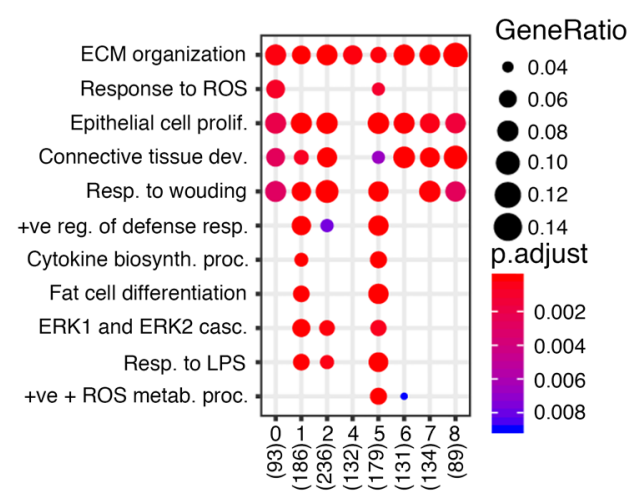

C

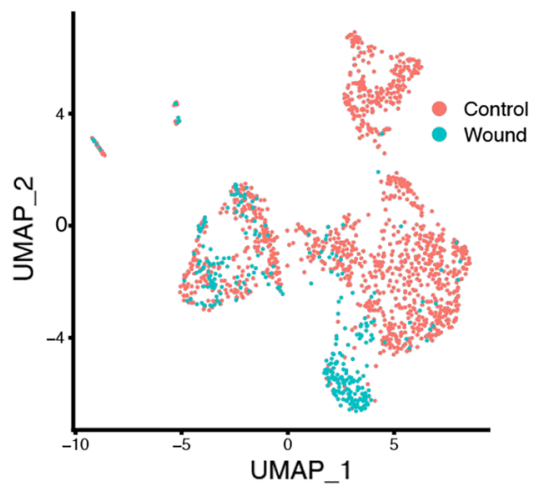

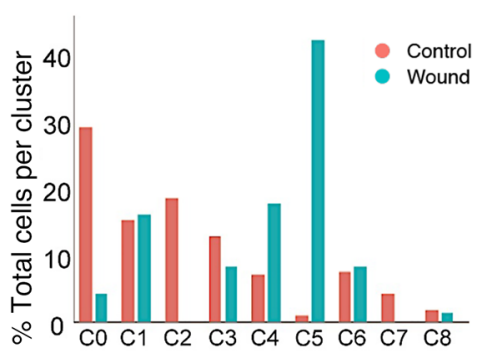

F

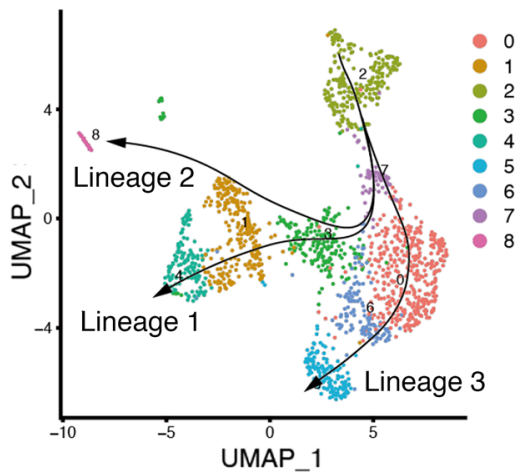

Marker genes in fat cell differentiation GO term

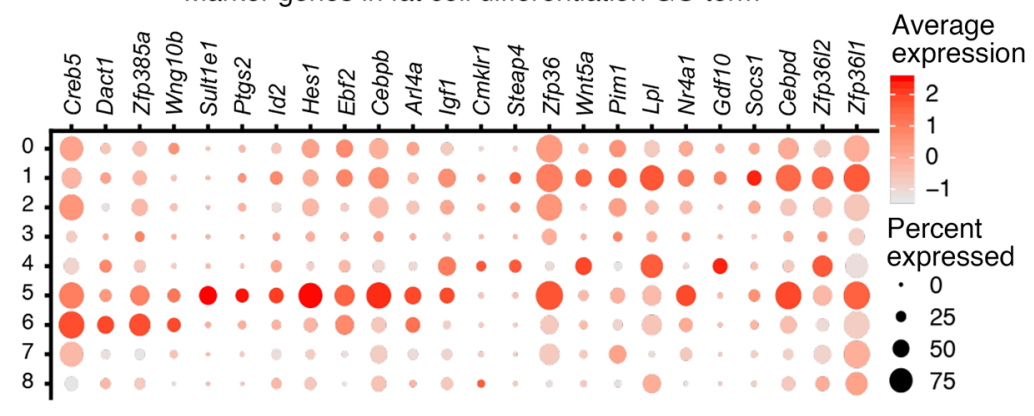

J

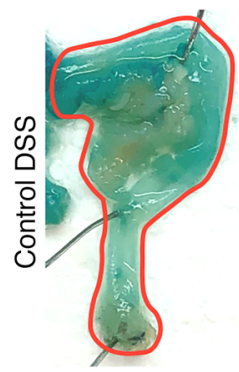

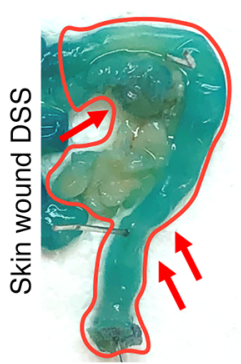

$\mathbf{K}$

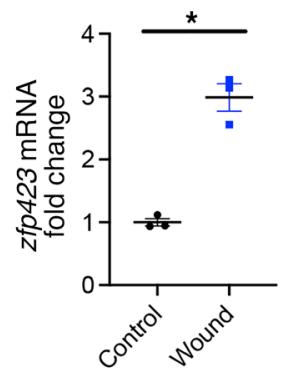

Figure 3. Skin injury alters the transcriptional profile of fibroblast subsets in the colon. UMAP plots of PDGFRA-positive cells from colon of mice with or without a skin wound, colored by gene cluster (A) and presence of a skin wound (B). (C) Relative abundance of each colon fibroblast cluster. (D and $\mathbf{E}$ ) Selected $\mathrm{CO}$ terms showing enrichment among genes by group (D) and cluster (E). resp., respiratory; cytok biosynth. proc., cytokine byosynthetic process; pep.-Tyr P04-lation, peptidyl-tyrosine phosphorylation; dev., development; casc., cascade. (F) Pseudotime analysis projected on UMAP plot. (G) Expression of marker genes as a function of pseudotime. (H) Expression of adipogenesis markers segregated across fibroblast clusters identified by scRNA-Seq. (I) Violin plots in fibroblast clusters $0-8$ for the expression of vimentin (Vim), a general fibroblast marker, and other genes as indicated. (J) $\beta$-Gal staining (blue) of Zfp423 4 lacZl+ mouse colon after DSS administration with or without skin wounding. Red arrows indicate areas of greatest increase in blue staining. (K) Expression of Zfp423 after DSS-induced colitis on day 7 with or without skin injury. ${ }^{*} P<0.05, t$ test; $n=3$. Error bars indicate mean $\pm \mathrm{SEM}$. 
A
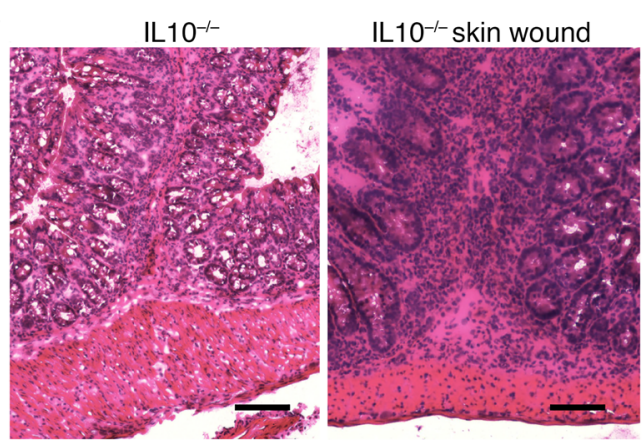

B

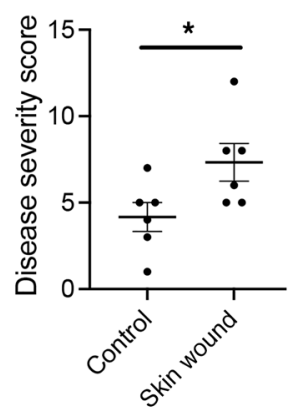

C

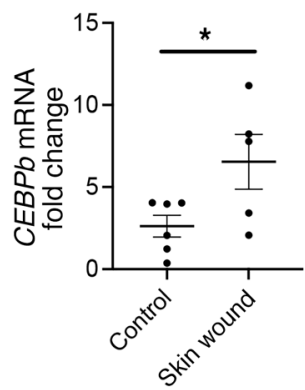

D
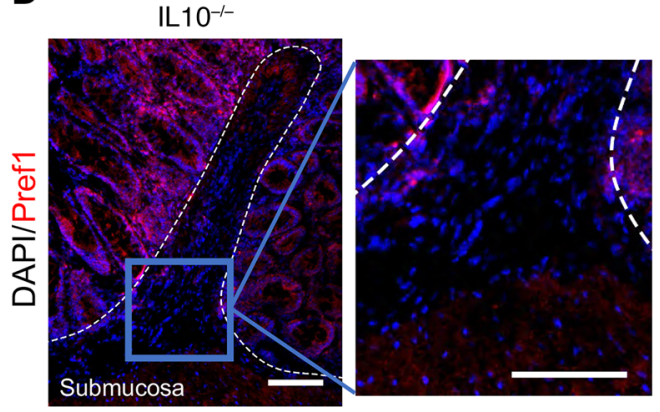

IL10-1- skin wound

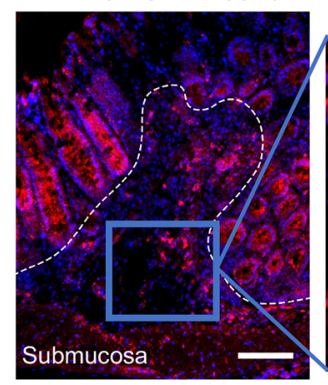

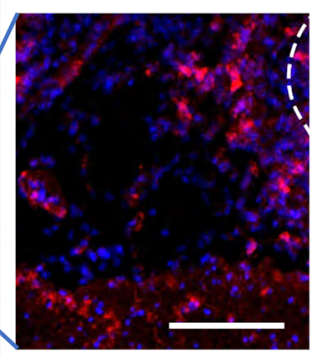

Figure 4. Histological evidence indicates that skin inflammation exacerbates colitis in IL10 ${ }^{-/-}$mice. IL10 ${ }^{-/-}$mice received incisional wounds on the back skin and colons were evaluated 2 days after the skin injury. (A) Histological images of colon from mice with or without skin wounds on day 2 . Tissue was stained with H\&E. (B) Disease activity score based on histological assessment of the colon. (C) mRNA expression of Cebpb in the colon on day 2 after skin wounding compared with that of uninjured controls $(n=6)$. (D) Representative images of mouse colon stained with Pref1 (red) in IL10 $0^{-/-}$mice on day 2 after skin wounding compared with uninjured controls. Dashed line delineates mucosa from submucosal region. Scale bars: $50 \mu \mathrm{m}$. Error bars indicate mean \pm SEM. ${ }^{*} P<0.05, t$ test; $n=6$. Each experiment was repeated 3 times.

findings demonstrate that in a setting where the local extracellular matrix remains intact, additional fragments of HA released from the skin serve as a DAMP, enhancing the potential of preadipocytes in the submucosa of the colon to respond to local injury. As the response to HA fragments and intestinal bacteria both involve TLR4, the 2 signals likely participate in the amplified response. This may prime the host defense system of the gut epithelial surface to be prepared for challenges first detected in the skin. However, as with all inflammatory responses, this can become a double-edged sword if the response is exaggerated or uncontrolled. Since these observations are consistent with clinical aspects of disease in IBD patients, blocking reactive adipogenesis or immune crosstalk by HA may be a therapeutic target. Overall, the data presented here show how the function of the skin should be considered when evaluating the function of other epithelial organs such as the gut.

\section{Methods}

Animals and animal care. For all animal studies, animals were randomly selected without formal prerandomization, and quantitative measurements were done were done in an unbiased manner.

Transgenic mice with conditional overexpression of hHYAL1 (in a C57BL/6 background) were generated in our laboratory as described previously by combining a constitutive promoter and a loxP-flanked GFP reporter upstream of Hyal1. WT (C57BL/6) and K14-cre-Tg, KikGR, CD44-KO, TLR2-KO, TLR4-KO, and IL10-KO mice were obtained from The Jackson Laboratory. In Zfp423 $3^{\text {lacz/+ }}$ mice (a gift from Maksim V. Plikus, University of California, Irvine, Irvine, California, USA), one allele of Zfp 423 gene has been inserted with a $\beta$-geo gene trap reporter, leading to the expression of a ZFP423-ßgeo fusion protein (46).K14-cre-Tg mice were bred with Hyal1 mice for the generation of K14-cre Hyal1 mice. K14-cre littermate controls were used in all experiments.

Bacterial strains. S. aureus strain USA300, a predominant community-associated methicillin-resistant $S$. aureus (MRSA) strain, and AH4807, a USA300 MRSA strain, were tested in a manner similar to that previously described $(47,48)$ and were provided by Alexander Horswill (Department of Immunology \& Microbiology, University of Colorado, Aurora, Colorado, USA).

Mouse model of DSS colitis. WT (C57BL/6), Zfp423 $23^{\text {lacZ/+ }}$, or K14-cre Hyal1 mice were provided 3\% DSS in drinking water for 7 days, and body weight was measured every day. Mice were sacrificed at the time points indicated. Histological severity was evaluated by disease activity index as described previously (9).

Mouse model of S. aureus skin infection and wounding. Skin infection experiments were done as described previously (49). S. aureus strain USA300 was used for infection. In brief, backs of sex-matched and age-matched (8-12 weeks) adult WT mice were shaved, with hair was removed by chemical depilation (Nair, Church \& Dwight), then injected subcutaneously with $100 \mu \mathrm{L}$ S. aureus at the mid-logarithmic growth phase $\left(2 \times 10^{6} \mathrm{CFU}\right.$ bacteria) in PBS. Forty-eight hours later, mice were provided 3\% DSS in drinking water for 7 days. Skin wound experiments were done as described previously (50). In brief, backs of sex-matched and age-matched (8-12 weeks) adult WT mice were 
A
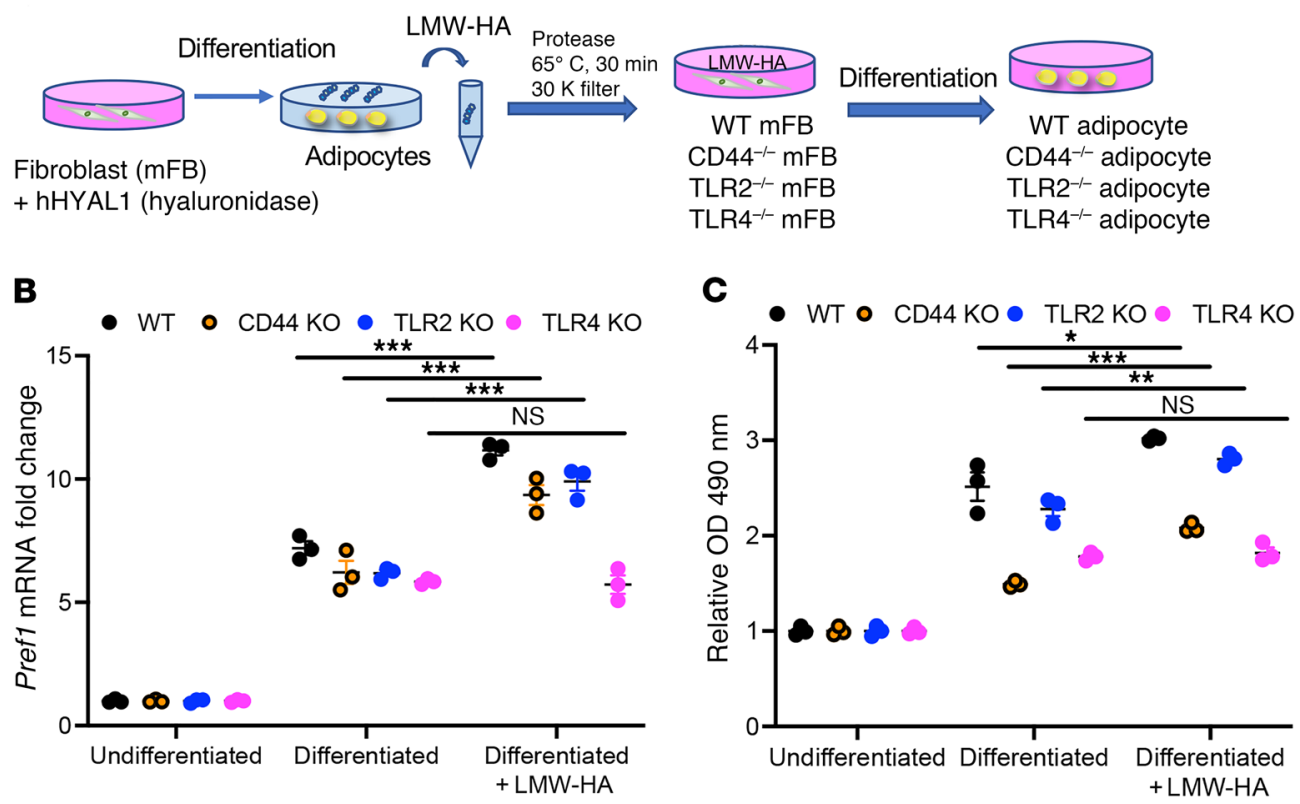

D

WT differentiated

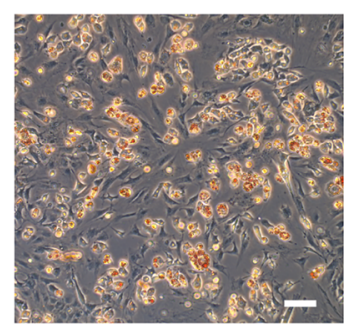

TLR4 $^{-1-}$ differentiated

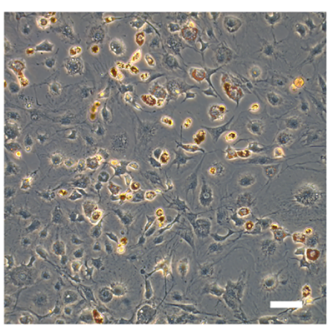

WT differentiated + LMW-HA

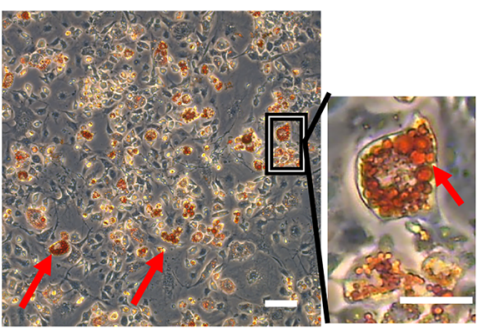

$\mathrm{TLR}^{-/-}$differentiated + LMW-HA

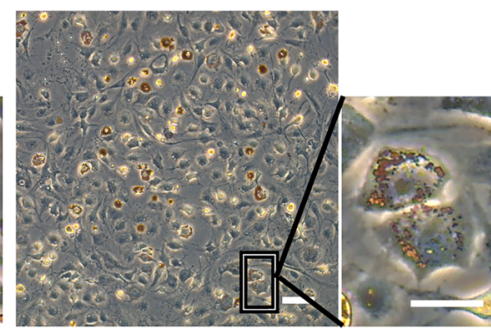

Figure 5. Adipogenic activation in vitro by HA through TLR4. (A) Illustration of the experimental design in which hHYAL1 was expressed in cultured fibroblasts to generate HA fragments for transfer to differentiating murine fibroblast preadipocytes (mFB). (B) Expression of mRNA for Pref1 in the mFBs on day 2. (C and $\mathbf{D})$ Microscopic images of colon mFBs cultured in conditions as indicated; cells were stained by Oil red 0 on day 6 . (C) Relative amount of Oil red $\mathrm{O}$ normalized by each undifferentiated condition. (D) Microscopic images of colon mFBs. Red arrows indicate areas of lipid droplet stained by Oil red 0. $n=3$. Scale bars: $50 \mu \mathrm{m}$. Error bars indicate mean \pm SEM. ${ }^{*} P<0.05,{ }^{* *} P<0.01,{ }^{* * *} P<0.001,2$-way ANOVA with correction for multiple comparisons. $n=3$ in all experiments, and each experiment was repeated 3 times.

shaved, with hair removed by chemical depilation (Nair), and were then given a $2 \mathrm{~cm}$ full-thickness incisional wound. Forty-eight hours later, mice were provided 3\% DSS in drinking water for 7 days.

Mouse model of IL10 $10^{-/}$colitis. IL10 ${ }^{-/-}$mice were obtained from The Jackson Laboratory at the age of 8 weeks. Mice were given a full-thickness incisional wound. Forty-eight hours later, skin and colon were harvested. Histology was evaluated by disease severity score as described previously (51).

Photoconversion procedures. Photoconversion procedures were adapted from methods developed previously (33). Mice were shaved and hair was removed. A silver LED 415 (Prizmatix) - set to maximum intensity, with a high-numerical-aperture polymer optical fiber (core diameter, $1.5 \mathrm{~mm}$ ) light guide and fiber collimater - was used as a 415 $\mathrm{nm}$ violet light source. After photoconversion for 10 minutes on the back, the irradiated skin site was wounded. Skin and colon tissue was collected 48 hours after injury. Flow cytometry was used to analyze skin and colon samples as previously described (52).

Whole mount lacZ staining. The colon was harvested at 7 days after DSS treatment from Zfp $423^{\text {lacz/+ }}$ mice and incubated with the X-gal reagent in LacZ staining buffer at $37^{\circ} \mathrm{C}$ overnight. This method has been described previously (51).

Reverse transcription quantitative PCR analyses. Reverse transcription quantitative PCR (RTqPCR) was used to determine mRNA abundance as described previously (53). Total cellular RNA was extracted using the PureLink RNA Mini Kit (Life Technologies) and mRNA was purified by use of Dynabeads mRNA Purification Kit (Life Technologies). mRNA (100 ng) was reverse transcribed to cDNA using the iScript cDNA Synthesis Kit (Bio-Rad). Quantitative real-time PCR was performed on the CFX96 real-time system (Bio-Rad) using a predeveloped TaqMan gene expression assay (Applied Biosystems). Expression of the housekeeping gene $\beta$-actin was used to normalize data.

Histology and immunohistochemistry. Tissue biopsy samples were directly embedded in OCT compound or paraffin. Paraffinembedded tissues were used for H\&E staining, and frozen sections were fixed in 4\% PFA for 20 minutes for immunofluorescence staining. For immunohistochemistry (IHC), fixed and permeabilized frozen tissue sections were blocked with Image-iT FX reagent (Invitrogen) before incubation with primary antibodies, followed by 
A

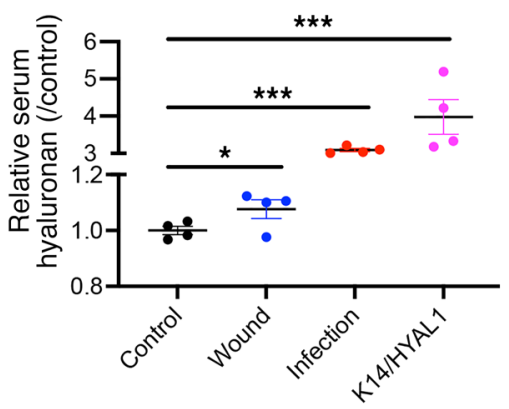

B

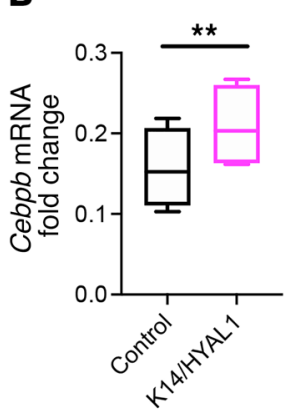

C

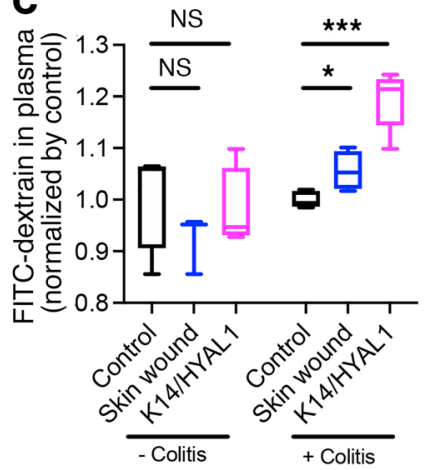

D

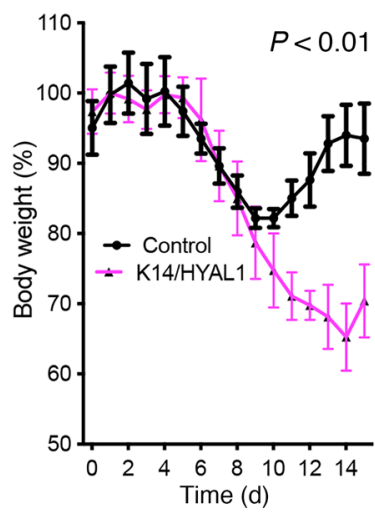

H

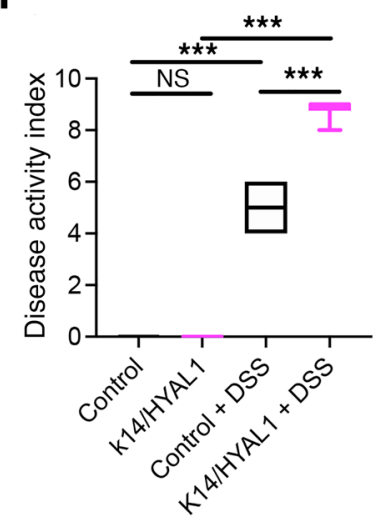

E

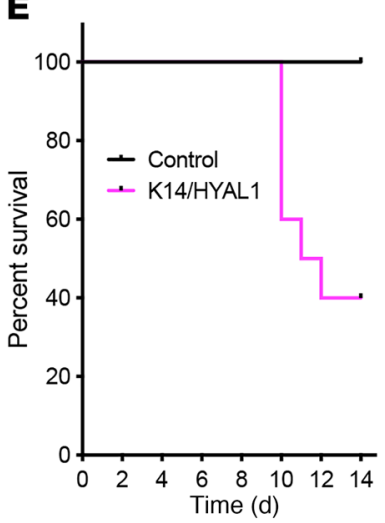

I

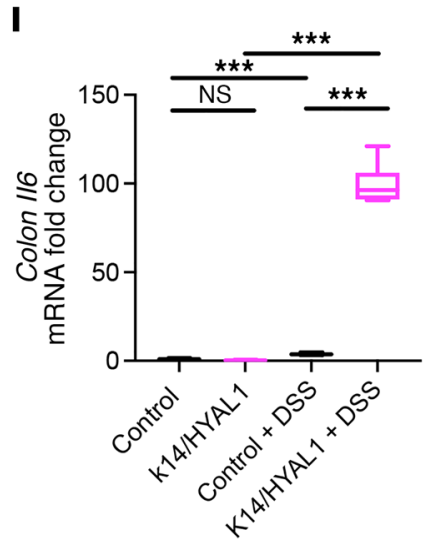

$\mathbf{F}$
Control DSS

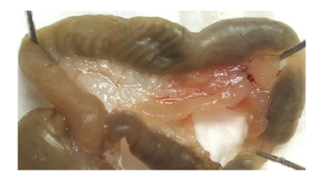

K14/HYAL1 DSS

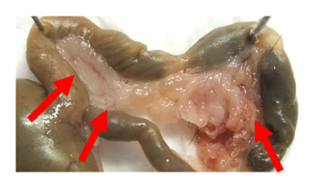

G

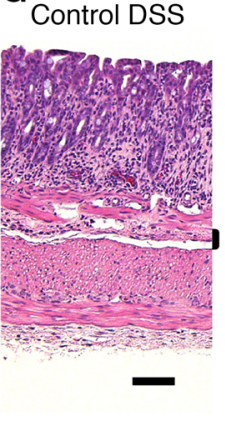

K14/HYALI1 DSS

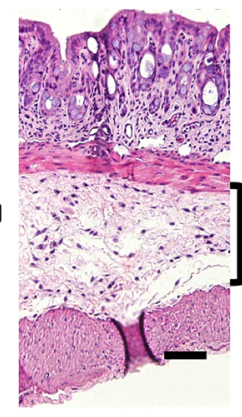

J

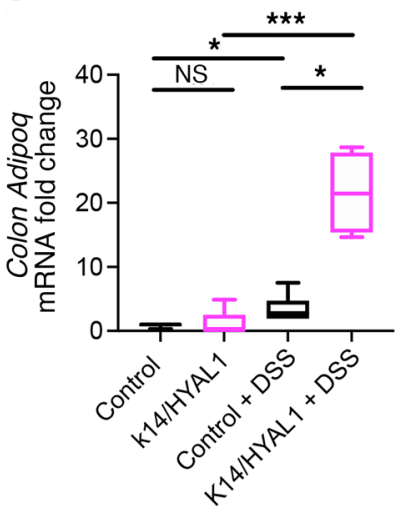

Figure 6. Expression of hyaluronidase in skin exacerbates sensitivity of the colon to DSS. (A) Relative abundance of HA in serum normalized by levels in control mice $(n=4)$. (B) Expression of Cebpb in the colon on day $O(n=6)$. (C) FITC-dextran assessment of intestinal permeability $(n=3)$. (D) Percent change in body weight normalized to weight on day $O(n=8)$. (E) Survival rate $(n=10)$. (F) Representative anatomical images of colon. Red arrows indicate areas of greatest increase in adipose tissue. (C) Histological images of the distal colon. Tissue stained with H\&E. Scale bars: $50 \mu \mathrm{m}$. (H) Disease activity index based on histological images of colon from mice on day $14(n=8)$. (I and J) Expression of $1 / 6$ and Adipoq in the colon $(n=8)$. Error bars indicate mean $\pm \mathrm{SEM} ;{ }^{*} P<0.05,{ }^{* *} P<0.01,{ }^{* * *} P<0.001$. In $\mathbf{A}$ and $\mathbf{B}, P$ values were based on $t$ test. In $\mathbf{C}, \mathbf{D}$, and $\mathbf{H}-\mathbf{J}, P$ values were based on 2 -way ANOVA with correction for multiple comparisons. Each experiment was repeated 3 times.

appropriate Alexa Fluor 488- or 568-coupled secondary antibodies. Nuclei were counterstained with DAPI. All images were taken with an Olympus BX41 microscope (widefield) or Zeiss LSM510 confocal microscope, as indicated.

$H A$ analysis. Glycosaminoglycans (GAGs), including HA, were extracted from mouse fibroblast culture supernatant as previously described (35). Samples were homogenized and treated overnight with protease $(0.16 \mathrm{mg} / \mathrm{mL}$; Sigma-Aldrich) to degrade protein, followed by purification by anion exchange chromatography using DEAE
Sephacel (Amersham Biosciences). Columns were washed with a lowsalt buffer ( $0.15 \mathrm{M} \mathrm{NaCl}$ in $20 \mathrm{mM}$ sodium acetate; $\mathrm{pH}$ 6.0) and eluted with $2 \mathrm{M} \mathrm{NaCl}$. Glycans were desalted by PD10 (GE Healthcare). The size distribution of HA was analyzed by agarose gel electrophoresis (54). The HA sample was mixed with Tris acetate EDTA (TAE) buffer containing $2 \mathrm{M}$ sucrose and electrophoresed at $2 \mathrm{~V} / \mathrm{cm}$ for 10 hours at room temperature. The gel was stained overnight under lightprotective cover at room temperature in a solution containing $0.005 \%$ Stains-All (Sigma-Aldrich) in 30\% ethanol. Serum for HA analysis was 
separated from mouse blood with a Serum separator additive tube (BD). HA concentrations were measured by an ELISA Duo Set (R\&D Systems). Defined HA size fractions of 6.8, 50, 150, 250, 500, and 1000 kDa were purchased from Seikagaku, Kogyo).

In situ hybridization. Fresh frozen skin and colon tissue sections were obtained from naive WT mice. Sections were stained for Krt14 using the RNAscope Fluorescent Multiplex Assay (Advanced Cell Diagnostics) according to the manufacturer's protocol with a predesigned Krt14 probe (catalog 422521) and the Amp4 Alt C color module.

RNA-Seq. Purified RNA from colon lamina propria or skin was submitted to the UCSD Institute for Genomic Medicine Core facility for library preparation and high-throughput next-generation sequencing for RNA (RNA integrity number $[\mathrm{RIN}]>9$ ). Libraries were constructed using TruSeq Stranded mRNA Library Prep Kits (Illumina) and run on a HiSeq 2500 instrument (Illumina). Raw data were analyzed using Partek Flow and Partek Genomics Suite software to determine transcript abundance and genes differentially expressed between samples. Gene Ontology analysis was performed using Metascape (http:// metascape.org).

Chemicals and reagents. HA-binding protein was purchased from Millipore. PREF1 antibody was purchased from GeneTex (GTX60309). Pref1, Adipoq, Pdgfra, Camp, and Il6 TaqMan gene expression assays were purchased from Life Technologies.

Microbiome analysis. Fecal samples were collected from cohoused control (WT) and K14/ HYAL1 mice under normal conditions, after 7 days of DSS treatment as the inflammatory condition and after 14 days as the recovered condition.

16S rRNA gene sequencing. DNA extraction and 16S rRNA amplicon sequencing were done using Earth Microbiome Project (EMP) standard protocols (http://www.earthmicrobiome.org/ protocols-and-standards/16s). DNA was extracted with the QIAGEN MagAttract PowerSoil DNA Kit as previously described (55). Amplicon PCR was performed on the V4 region of the 16S rRNA gene using the primer pair 515f to $806 \mathrm{r}$ with Golay error-correcting barcodes on the reverse primer. Amplicons were barcoded and pooled in equal concentrations for sequencing. The amplicon pool was purified with the MO BIO UltraClean PCR Cleanup Kit and sequenced on the Illumina MiSeq sequencing platform. Sequence data were demultiplexed and minimally quality filtered, trimmed to 150 bases, and processed using Deblur v1.0.4 (56) using the Qiita default parameters (i.e., setting min-reads 1) to generate suboperational taxonomic units (sOTUs). Taxonomies for sOTUs were assigned using the sklearn-based taxonomy classifier trained on the Greengenes reference database 13_8 (57) clustered at 99\% similarity (feature classifier plug-in of QIIME 2 v2019.1; ref.58). The sOTU table was rarefied to a depth of 5000 sequenchttps:// onlinelibrary.wiley.com/doi/10.1002/9781118445112.stat07841\#es/ sample to control for sequencing effort (59). The deblurred sequence fragments were inserted into the Greengenes $13 \_8$ phylogenetic tree using SATé-enabled phylogenetic placement $(60,61)$.

$16 S$ marker gene data analysis. QIIME 2 v. 2018.8 (61) was used to generate pairwise unweighted UniFrac distances (62). Between-group differences based on these distances were tested using PERMANOVA (63) and permuted $t$ tests in QIIME 2. principal coordinates analysis results were visualized in EMPeror (56).

scRNA-Seq. Tissue samples from 3 mice in each group were minced with a razor blade into $1 \mathrm{~cm}$ fragments, suspended in enzymatic digestion buffer containing collagenase and DNase I as previ- ously described (64), incubated with frequent agitation at $37^{\circ} \mathrm{C}$ for 30 minutes, and triturated briefly with a $5 \mathrm{~mL}$ pipet. Cells in a single-cell suspension were then passed through a $100 \mu \mathrm{m}$ mesh filter, centrifuged, and stained with a live/dead stain and anti-PDGFRA antibodies for FACS sorting for cells of fibroblast lineage. 20,000 sorted cells were loaded on the 10x Genomics Chromium system.

Library construction protocol. Single-cell suspensions were loaded onto the 10x Genomics Chromium Controller instrument to generate single-cell Gel Bead-in-Emulsion (GEMs). GEM-RT and library construction were performed following the 10x Genomics protocol. Library fragment size distributions were determined using an Agilent Bioanalyzer High Sensitivity chip, and library DNA concentrations were determined using a Qubit 2.0 Fluorometer (Invitrogen). Libraries were sequenced using an Illumina HiSeq4000.

Data analysis. For mouse colonfibroblasts, the $10 \mathrm{x}$ Genomics Cell Ranger version 3.0.1 software pipeline with default parameters was used to perform sample demultiplexing, barcode processing, alignment to the $\mathrm{mm} 10$ reference genome, and single-cell gene counting. Data were further filtered, processed, and analyzed using the Seurat $\mathrm{R}$ toolkit version $3(65,66)$. Filtering of initial data involved selecting cells with $>200$ and $<4000$ features and $<7.5 \%$ mitochondrial genes. Data were normalized using the NormalizeData() function, with parameters normalization.method = "LogNormalize" and scale. factor $=10000$. Variable genes were identified with the FindVariableFeatures() function, with parameters with selection.method = "vst" and nfeatures = 2000. Data were scaled with the ScaleDa$\mathrm{ta}()$ function, with parameters vars.to.regress = "percent.mt" and "nUMI". Principal components were calculated from these variable genes using the function RunPCA(). Selection of significant principal components (PCs) to include in further analyses was determined using the JackStraw() method with the num.replicate parameter set to 100 , along with visualization of each PC using the DimHeatmap() function. Significant PCs $(1: 13,15,16$, and 18) were then selected, and clusters were identified using the FindNeighbors() function using the significant PCs, then the FindClusters() function with parameter resolution $=0.5$. Nonlinear dimensionality reduction and visualization was performed with tSNE using the RunTSNE(), and UMAP (67) using the RunUMAP() function. Marker genes for clusters and between samples were determined using the FindAllMarkers() function, with parameters min.pct $=0.25$ and thresh.use $=0.25$. Gene ontology analysis was performed on marker genes using the "clusterProfiler" R package with default parameters (68). Cell lineage and pseudotime inference on mouse colon fibroblasts was performed using the Slingshot R package (27). The Seurat object was transformed into a SingleCellExperiment object, and Slingshot trajectory analysis was performed in an unsupervised manner using Seurat clustering information and uniform manifold approximation and projection (UMAP) dimensionality reduction. For human colon fibroblasts, data were downloaded from the NCBI's Gene Expression Omnibus database (GEO GSE114374) (23). The Health and UC datasets were merged into a single Seurat object using the Seurat merge() function. Marker genes were identified using the FindAllMarkers() function, with parameters test.use = "LR", latent.vars $=$ "Exp", min.pct $=0.25$, and logfc.threshold $=0.4054651$ (corresponding to 1.5 -fold change). For UMAP projections, the Health and UC data sets were first integrated using FindIntegrationAnchors(), IntegrateData() as described in the Standard Workflow described 
in the Seurat Integration and Label Transfer tutorial and in ref. 66. Data were scaled and PCs calculated as described above. The first 30 PCs were used for UMAP projections.

Flow cytometry. Colon tissue collected from control or K14/ HYAL1 mice was cut into small pieces, then digested with $2.5 \mathrm{mg} /$ $\mathrm{mL}$ Collagenase D and $30 \mathrm{ng} / \mathrm{mL}$ DNase I for 2 hours at $37^{\circ} \mathrm{C}$, then filtered through a $70 \mu \mathrm{m}$ filter to generate single-cell suspension for FACS analyses. Cells were then blocked with anti-mouse CD16/32 (eBioscience, 14016185), followed by staining with an antibody cocktail for immune cells. The antibody cocktail for immune cells included PE/cy7-CD11b (BioLegend, 101216), Per/cy5.5-Ly6G (eBioscience, 11593182), PE-F4/80 (eBioscience,12480182), APC-CD11c (BioLegend, 117310), AF700-MHCII (eBioscience, 56532182), APCe780-CD4 (Fisher Scientific, 47004282), BV650-CD8a (BioLegend, 100741), and Fixable Viability Dye (eBioscience, 65-0866-14). FACS analyses for surface expression of preadipocyte or immune cell markers were performed by the BD FACSCanto RUO machine and data analyzed by FlowJo V10 software. Dead cells that stained positive with Viability Dye were excluded from the analyses.

Statistics. Experiments were repeated at least 3 times, with similar results. Statistical significance was determined using Student's unpaired 2-tailed $t$ test, or 1-way ANOVA multiple-comparison test, as indicated in the legends. $P$ values less than 0.05 were considered significant.
Data availability. All data have been deposited in the GEO database (GSE158859).

Study approval. All animal experiments were approved by the UCSD Institutional Animal Care and Use Committee.

\section{Author contributions}

TD conceptualized the study, performed investigations, and wrote the original draft of the manuscript; MCL, JSS, BCT, KJC, FL, NHS, and JO designed and performed experiments and/or analyzed and interpreted results. RK, JC, and NHS supervised the study; RLG conceptualized and supervised the study and wrote the manuscript.

\section{Acknowledgments}

Christopher Glass provided computing resources for the scRNASeq analysis. KJC is supported by NIH grant T32 DK007202; RLG and NHS are supported by NIH R01DK121760. RLG is additionally supported by NIH R37AI052453, R01AR076082, and R01AR069653.

Address correspondence to: Richard L. Gallo, Department of Dermatology, MC0869, University of California, San Diego, 9500 Gilman Dr, La Jolla, California 92093-0869, USA. Phone: 858.822.4608; Email: rgallo@ucsd.edu.
1. Vavricka SR, et al. Frequency and risk factors for extraintestinal manifestations in the Swiss inflammatory bowel disease cohort. Am J Gastroenterol. 2011;106(1):110-119.

2. Vlachos C, et al. Psoriasis and inflammatory bowel disease: links and risks. Psoriasis (Auckl). 2016;6:73-92

3. Fimmel S, Zouboulis CC. Comorbidities of hidradenitis suppurativa (acne inversa). 2010;2(1):9-16.

4. Kredel LI, Siegmund B. Adipose-tissue and intestinal inflammation - visceral obesity and creeping fat. Front Immunol. 2014;5:462.

5. Kim M, et al. Inflammatory bowel disease is associated with an increased risk of inflammatory skin diseases: a population-based cross-sectional study. J Am Acad Dermatol. 2017;76(1):40-48.

6. Wu CY, et al. Risk of inflammatory bowel disease in patients with rosacea: results from a nationwide cohort study in Taiwan. JAm Acad Dermatol. 2017;76(5):911-917.

7. Muraro A, et al. Precision medicine in patients with allergic diseases: airway diseases and atopic dermatitis-PRACTALL document of the European Academy of Allergy and Clinical Immunology and the American Academy of Allergy, Asthma \& Immunology. J Allergy Clin Immunol. 2016;137(5):1347-1358.

8. Gallo RL, Hooper LV. Epithelial antimicrobial defence of the skin and intestine. Nat Rev Immunol. 2012;12(7):503-516.

9. Dokoshi T, et al. Hyaluronidase inhibits reactive adipogenesis and inflammation of colon and skin. JCI Insight. 2018;3(21):e123072.

10. Ha CWY, et al. Translocation of viable gut microbiota to mesenteric adipose drives formation of creeping fat in humans. Cell. 2020;183(3):666-683.

11. Mao R, et al. Activated intestinal muscle cells promote preadipocyte migration: a novel mech- anism for creeping fat formation in Crohn's disease [published online January 19, 2021]. Gut. https://doi.org/10.1136/gutjnl-2020-323719.

12. Zhang LJ, et al. Innate immunity. Dermal adipocytes protect against invasive Staphylococcus aureus skin infection. Science. 2015;347(6217):67-71.

13. Lackey DE, Olefsky JM. Regulation of metabolism by the innate immune system. Nat Rev Endocrinol. 2016;12(1):15-28.

14. McLaughlin T, et al. Role of innate and adaptive immunity in obesity-associated metabolic disease. J Clin Invest. 2017;127(1):5-13.

15. Ji E, et al. Inhibition of adipogenesis in 3T3-L1 cells and suppression of abdominal fat accumulation in high-fat diet-feeding C57BL/6J mice after downregulation of hyaluronic acid. Int JObes (Lond). 2014;38(8):1035-1043

16. Chen PY, et al. Hyaluronan preserves the proliferation and differentiation potentials of long-term cultured murine adipose-derived stromal cells. Biochem Biophys Res Commun. 2007;360(1):1-6.

17. Jiang D, et al. Hyaluronan as an immune regulator in human diseases. Physiol Rev 2011;91(1):221-264.

18. Schommer NN, et al. Hyaluronan breakdown contributes to immune defense against group A Streptococcus. J Biol Chem. 2014;289(39):26914-26921.

19. Garantziotis S, Savani RC. Hyaluronan biology: a complex balancing act of structure, function, location and context. Matrix Biol. 2019;78-79:1-10.

20. Muto J, et al. Emerging evidence for the essential role of hyaluronan in cutaneous biology. J Dermatol Sci. 2019;94(1):190-195.

21. Foell D, et al. Mechanisms of disease: a 'DAMP' view of inflammatory arthritis. Nat Clin Pract Rheumatol. 2007;3(7):382-390.
22. Kolar SL, et al. Group B streptococcus evades host immunity by degrading hyaluronan. Cell Host Microbe. 2015;18(6):694-704.

23. Kinchen J, et al. Structural remodeling of the human colonic mesenchyme in inflammatory bowel disease. Cell. 2018;175(2):372-386.

24. Guerrero-Juarez CF, et al. Single-cell analysis reveals fibroblast heterogeneity and myeloidderived adipocyte progenitors in murine skin wounds. Nat Commun. 2019;10(1):650.

25. Grada A, et al. Research techniques made simple: animal models of wound healing. J Invest Dermatol. 2018;138(10):2095-2105.

26. Chassaing B, et al. Dextran sulfate sodium (DSS)-induced colitis in mice. Curr Protoc Immunol. 2014;104:15.25.1-15.25.14.

27. Street K, et al. Slingshot: cell lineage and pseudotime inference for single-cell transcriptomics. BMC Genomics. 2018;19(1):477.

28. Park K, et al. Regulation of cathelicidin antimicrobial peptide expression by an endoplasmic reticulum (ER) stress signaling, vitamin D receptor-independent pathway. J Biol Chem. 2011;286(39):34121-34130.

29. Gupta RK, et al. Transcriptional control of preadipocyte determination by Zfp423. Nature. 2010;464(7288):619-623.

30. Harder L, et al. ZNF423: transcriptional modulation in development and cancer. Mol Cell Oncol. 2014;1(3):e969655.

31. Gulyaeva O, et al. Sox9-meis1 inactivation is required for adipogenesis, advancing Pref- $1^{+}$to PDGFR $\alpha^{+}$cells. Cell Rep. 2018;25(4):1002-1017.

32. Sawada Y, et al. Resolvin E1 inhibits dendritic cell migration in the skin and attenuates contact hypersensitivity responses. J Exp Med. 2015;212(11):1921-1930.

33. Shand FH, et al. Tracking of intertissue 
migration reveals the origins of tumorinfiltrating monocytes. Proc Natl Acad Sci U S A. 2014;111(21):7771-7776.

34. Dokoshi $T$, et al. Hyaluronan degradation by cemip regulates host defense against staphylococcus aureus skin infection. Cell Rep. 2020;30(1):61-68.

35. Muto J, et al. Hyaluronan digestion controls DC migration from the skin. JClin Invest. 2014;124(3):1309-1319.

36. Wang C, et al. Hyaluronan distribution in the normal epithelium of esophagus, stomach, and colon and their cancers. Am J Pathol. 1996;148(6):1861-1869.

37. Pandey MS, et al. The cytoplasmic domain of the hyaluronan receptor for endocytosis (HARE) contains multiple endocytic motifs targeting coated pit-mediated internalization. J Biol Chem. 2008;283(31):21453-21461.

38. Foley JP, et al. Toll-like receptor 2 (TLR2), transforming growth factor- $\beta$, hyaluronan (HA), and receptor for HA-mediated motility (RHAMM) are required for surfactant protein A-stimulated macrophage chemotaxis. J Biol Chem. 2012;287(44):37406-37419.

39. Mohamadzadeh M, et al. Proinflammatory stimuli regulate endothelial hyaluronan expression and CD44/HA-dependent primary adhesion. JClin Invest. 1998;101(1):97-108.

40. Taylor KR, et al. Hyaluronan fragments stimulate endothelial recognition of injury through TLR4. JBiol Chem. 2004;279(17):17079-17084.

41. Jiang D, et al. Regulation of lung injury and repair by Toll-like receptors and hyaluronan. Nat Med. 2005;11(11):1173-1179.

42. Leu SW, et al. TLR4 through IFN- $\beta$ promotes low molecular mass hyaluronan-induced neutrophil apoptosis. JImmunol. 2011;186(1):556-562.

43. Liang J, et al. Hyaluronan and TLR4 promote surfactant-protein-C-positive alveolar progenitor cell renewal and prevent severe pulmonary fibrosis in mice. Nat Med.2016;22(11):1285-1293.
44. Misra S, et al. Interactions between hyaluronan and its receptors (CD44, RHAMM) regulate the activities of inflammation and cancer. Front Immunol. 2015;6:201.

45. Ghatak S, et al. Roles of proteoglycans and glycosaminoglycans in wound healing and fibrosis. Int JCell Biol. 2015;2015:834893.

46. Cheng LE, et al. The transcription factor Zfp423/OAZ is required for cerebellar development and CNS midline patterning. Dev Biol. 2007;307(1):43-52.

47. Muhs A, et al. Virulence inhibitors from brazilian peppertree block quorum sensing and abate dermonecrosis in skin infection models. Sci Rep. 2017;7:42275.

48. Paharik AE, et al. Coagulase-negative staphylococcal strain prevents staphylococcus aureus colonization and skin infection by blocking quorum sensing. Cell Host Microbe. 2017;22(6):746-756

49. Nizet $V$, et al. Innate antimicrobial peptide protects the skin from invasive bacterial infection. Nature. 2001;414(6862):454-457.

50. Wong VW, et al. Surgical approaches to create murine models of human wound healing. JBiomed Biotechnol. 2011;2011:969618.

51. Ito M, et al. Wnt-dependent de novo hair follicle regeneration in adult mouse skin after wounding. Nature. 2007;447(7142):316-320.

52. Gray EE, et al. Deficiency in IL-17-committed $\mathrm{V} \gamma 4(+) \gamma \delta \mathrm{T}$ cells in a spontaneous Sox13-mutant CD45.1(+) congenic mouse substrain provides protection from dermatitis. Nat Immunol. 2013;14(6):584-592.

53. Morioka Y, et al. Cathelicidin antimicrobial peptides inhibit hyaluronan-induced cytokine release and modulate chronic allergic dermatitis. J Immunol. 2008;181(6):3915-3922.

54. Lee HG, Cowman MK. An agarose gel electrophoretic method for analysis of hyaluronan molecular weight distribution. Anal Biochem. 1994;219(2):278-287.

55. Marotz C, et al. DNA extraction for streamlined metagenomics of diverse environmental samples. Biotechniques. 2017;62(6):290-293.

56. Amir A, et al. Deblur rapidly resolves singlenucleotide community sequence patterns. mSystems. 2017;2(2):e00191-16.

57. McDonald D, et al. An improved Greengenes taxonomy with explicit ranks for ecological and evolutionary analyses of bacteria and archaea. ISME J. 2012;6(3):610-618.

58. Bolyen E, et al. Reproducible, interactive, scalable and extensible microbiome data science using QIIME 2. Nat Biotechnol. 2019;37(8):852-857.

59 . Weiss $\mathrm{S}$, et al. Normalization and microbial differential abundance strategies depend upon data characteristics. Microbiome. 2017;5(1):27.

60. Mirarab S, et al. SEPP: SATé-enabled phylogenetic placement. Pac Symp Biocomput. 2012:247-258.

61. Janssen S, et al. Phylogenetic placement of exact amplicon sequences improves associations with clinical information. mSystems. 2018;3(3):e00021-18.

62. Lozupone C, Knight R. UniFrac: a new phylogenetic method for comparing microbial communities. Appl Environ Microbiol. 2005;71(12):8228-8235.

63. Anderson MJ. Permutational Multivariate Analysis of Variance (PERMANOVA). Department of Statistics, University of Auckland; 2005. https://doi. org/10.1002/9781118445112.stat07841.

64 . Ohnmacht C. Tolerance to the intestinal microbiota mediated by ROR $(\gamma \mathrm{t})(+)$ cells. Trends Immunol. 2016;37(7):477-486.

65. Butler A, et al. Integrating single-cell transcriptomic data across different conditions, technologies, and species. Nat Biotechnol. 2018;36(5):411-420.

66. Stuart T, et al. Comprehensive integration of single-cell data. Cell. 2019;177(7):1888-1902.

67. Becht E, et al. Dimensionality reduction for visualizing single-cell data using UMAP. Nat Biotechnol. 2019;37:38-44.

68. Yu G, et al. clusterProfiler: an R package for comparing biological themes among gene clusters. OMICS. 2012;16(5):284-287. 